PROCEEDINGS OF THE

AMERICAN MATHEMATICAL SOCIETY

Volume 136, Number 8, August 2008, Pages 2763-2769

S 0002-9939(08)09476-8

Article electronically published on March 21, 2008

\title{
ON THE FULL REGULARITY OF THE FREE BOUNDARY IN A CLASS OF VARIATIONAL PROBLEMS
}

\author{
ARSHAK PETROSYAN
}

(Communicated by David S. Tartakoff)

ABSTRACT. We consider nonnegative minimizers of the functional

$$
J_{p}(u ; \Omega)=\int_{\Omega}|\nabla u|^{p}+\lambda_{p}^{p} \chi_{\{u>0\}}, \quad 1<p<\infty,
$$

on open subsets $\Omega \subset \mathbb{R}^{n}$. There is a critical dimension $k^{*}$ such that the free boundary $\partial\{u>0\} \cap \Omega$ has no singularities and is a real analytic hypersurface if $p=2$ and $n<k^{*}$. A corollary of the main result in this note ensures that there exists $\epsilon_{0}>0$ such that the same result holds if $|p-2|<\epsilon_{0}$.

\section{INTRODUCTION}

Let $\Omega$ be a bounded open set in $\mathbb{R}^{n}$ and $u \in W^{1, p}(\Omega) \cap L^{\infty}(\Omega)$ be a minimizer of the functional

$$
J_{p}(u ; \Omega)=\int_{\Omega}|\nabla u|^{p}+\lambda_{p}^{p} \chi_{\{u>0\}}, \quad 1<p<\infty,
$$

in the sense that $J_{p}(u ; \Omega) \leq J_{p}(v ; \Omega)$ for any $v \in u+W_{0}^{1, p}(\Omega)$. Here $\lambda_{p}$ is a positive constant. Everywhere in this paper we restrict ourselves to nonnegative minimizers $u$. Such minimizers solve the following one-phase Bernoulli-type free boundary problem:

$$
\begin{aligned}
& \Delta_{p} u:=\operatorname{div}\left(|\nabla u|^{p-2} \nabla u\right)=0 \text { in }\{u>0\}, \\
& |\nabla u|=q_{p} \text { on } \Gamma(u):=\partial\{u>0\} \cap \Omega
\end{aligned}
$$

in a certain weak sense, where $q_{p}=\lambda_{p}(p-1)^{-1 / p}$. We are then interested in the regularity properties of the free boundary $\Gamma(u)$.

This problem was first studied in a seminal paper of Alt and Caffarelli [AC81, in the case $p=2$, where they established the Lipschitz continuity of the minimizers as well as the following regularity result concerning the free boundary:

The measure-theoretic reduced boundary $\Gamma_{\text {red }}(u):=\partial_{\text {red }}\{u>0\} \cap$ $\Omega$ is locally an analytic hypersurface; moreover, the singular set $\Sigma(u):=\Gamma(u) \backslash \Gamma_{\text {red }}(u)$ has Hausdorff $(n-1)$-measure zero $H^{n-1}(\Sigma(u))$ $=0$.

Received by the editors March 6, 2006

2000 Mathematics Subject Classification. Primary 35R35.

Key words and phrases. Regularity of the free boundary, degenerate/singular variational problem, Bernstein-type theorem, improvement of flatness.

The author was supported in part by NSF grant DMS-0401179.

(C)2008 American Mathematical Society 
In the same paper, Alt and Caffarelli proved that in dimension $n=2$ (still the case $p=2$ ) the singular set $\Sigma(u)$ is empty, i.e. the free boundary is fully regular. More than twenty years later Caffarelli, Jerison and Kenig CJK04 were able to extend this result to $n=3$. They showed that there are no minimal cones (i.e. homogeneous of degree one minimizers of $J_{2}$ ) other than halfspace solutions

$$
u(x)=q_{p}(x \cdot e)^{+}, \quad x \in \mathbb{R}^{n},
$$

where $e$ is a unit vector in $\mathbb{R}^{n}$. (We use the notation $\alpha^{+}=\max \{\alpha, 0\}$.) The regularity of the free boundary then follows from the result of Weiss Wei99 based on a monotonicity formula and similar to that of minimal surface theory. In fact, Weiss also established the existence of a critical dimension $k^{*}$ with the following property:

For any minimizer $u$ of $J_{2}$, the singular set $\Sigma(u)$ is empty if $n<k^{*}$, consists of at most isolated points if $n=k^{*}$, and has Hausdorff dimension at most $n-k^{*}$ for $n \geq k^{*}$.

It is currently known that

$$
4 \leq k^{*} \leq 7 .
$$

The lower bound follows from CJK04, and the upper bound was recently established by De Silva and Jerison DSJ05.

The history of the problem is much shorter for $1<p<\infty$. In DP05, Danielli and the author have extended Alt and Caffarelli's result that $\Gamma_{\text {red }}(u)$ is locally an analytic hypersurface and that $H^{n-1}(\Sigma(u))=0$. Subsequently, in [DP06, it was established that in dimension 2 the singular set is empty for $p$ in the range $2-\epsilon_{0}<p<\infty$, where $\epsilon_{0}>0$ is an absolute constant. Further study of the problem is complicated because of the unavailability of a Weiss-type monotonicity formula, even in dimension $n=2$.

The main goal of this note is to show that despite the additional difficulties associated with $p \neq 2$, there is a simple limiting argument, combined with a uniform-in- $p$ "flatness implies regularity" theorem, that ensures that $\Sigma(u)$ is empty for $p$ in the range $2-\epsilon_{0}<p<2+\epsilon_{0}$ for some $\epsilon_{0}$ in any space dimension $n<k^{*}$. This simplifies and extends a similar argument in DP06] to higher dimensions.

Definition 1.1. Let $\mathcal{R}(n)$ be the set of exponents $p, 1<p<\infty$, such that any minimizer of $J_{p}$ on any open subset of $\mathbb{R}^{n}$ has no singular free boundary points.

In particular, we know that $2 \in \mathcal{R}(2), 2 \in \mathcal{R}(3)$, but $2 \notin \mathcal{R}(7)$; see above.

Theorem 1.2 (Main result). Let $p_{0} \in \mathcal{R}(n)$. Then there exists $\epsilon_{0}=\epsilon\left(p_{0}\right)>0$ such that $\left(p_{0}-\epsilon_{0}, p_{0}+\epsilon_{0}\right) \subset \mathcal{R}(n)$. In other words, $\mathcal{R}(n)$ is an open set.

With the critical exponent $k^{*}$ as defined above, we immediately obtain the following result.

Corollary 1.3. There exists $\epsilon_{0}>0$ such that if $2-\epsilon_{0}<p<2+\epsilon_{0}$ and $2 \leq n<k^{*}$, then for any minimizer $u$ of $J_{p}$ on an open subset of $\mathbb{R}^{n}$, the free boundary $\Gamma(u)$ is an analytic hypersurface.

The paper is organized as follows. In Section 2 we recall some known facts and results, including the Lipschitz regularity of minimizers, nondegeneracy, blowups with variable $p$, as well as the "flatness implies regularity" theorem. In Section 3 we prove a Bernstein-type theorem for global minimizers of $J_{p}$ and give the proof of the main result of this note (Theorem 1.2). 


\section{PREliminaries AND KNOWN RESUlts}

In this section we state without proof some known results on minimizers of $J_{p}$. The proofs can be found in [DP05] and [DP06]. For the locally uniform dependence of the constants on $p \in[1+\mu, 1+1 / \mu], 0<\mu<1$, we also refer to a recent paper by Martínez and Wolanski MW06, where they consider the minimizers of a more general functional

$$
\int_{\Omega} G(|\nabla u|)+\lambda_{G} \chi_{\{u>0\}}
$$

where $G$ is a power-like function satisfying $\mu \leq t G^{\prime \prime}(t) / G^{\prime}(t) \leq 1 / \mu$ for some $\mu>0$.

2.1. Scaling and blowups. Let $u$ be a minimizer of $J_{p}$ in $\Omega$ and $x_{0} \in \partial \Gamma(u)$. Since we are interested in local properties of the free boundary, without loss of generality we may assume that $\Omega=B_{\rho}\left(x_{0}\right)$. Moreover, dividing $u$ by the constant $q_{p}$ as in (1.2), without loss of generality we will assume that the constant $\lambda_{p}$ in (1.1) is normalized so that $q_{p}=1$.

Theorem 2.1 (Lipschitz continuity). Let $u$ be a minimizer of $J_{p}$ in $B_{\rho}\left(x_{0}\right)$ and $p \in[1+\mu, 1+1 / \mu], 0<\mu<1$. Then there exists a constant $C=C(n, \mu)>0$ such that

$$
|\nabla u| \leq C \quad \text { in } \quad B_{\rho / 2}\left(x_{0}\right) .
$$

Theorem 2.2 (Nondegeneracy). Let $u$ be a minimizer of $J_{p}$ in $B_{\rho}\left(x_{0}\right), x_{0} \in \Gamma(u)$, and $p \in[1+\mu, 1+1 / \mu], 0<\mu<1$. Then there exist $c=c(\mu, n)>0, \gamma=\gamma(\mu)>1$ such that

$$
\left(f_{B_{r}\left(x_{0}\right)} u^{\gamma}\right)^{1 / \gamma} \geq c r \quad \text { if } \quad B_{r / 2}\left(x_{0}\right) \cap\{u>0\} \neq \emptyset,
$$

for any $0<r \leq \rho / 2$.

For the minimizer $u$ as in Theorem 2.1 consider the rescalings

$$
u_{\lambda}(x)=u_{x_{0}, \lambda}(x)=\frac{u\left(\lambda x+x_{0}\right)}{\lambda}, \quad x \in B_{\rho / \lambda} .
$$

It is easy to see that $u_{\lambda}$ is a minimizer of the same functional $J_{p}$ in $B_{\rho / \lambda}$ and $0 \in \Gamma\left(u_{\lambda}\right)$. Moreover, by Theorem 2.1 .

$$
\left|\nabla u_{\lambda}\right| \leq C \quad \text { on } \quad B_{R}
$$

for $\lambda \leq \rho / 2 R$. So, if we let $\lambda \rightarrow 0$, from local equicontinuity, we can find a subsequence $\lambda=\lambda_{k} \rightarrow 0$ such that the rescalings $u_{\lambda_{k}}$ converge in $L_{\text {loc }}^{\infty}\left(\mathbb{R}^{n}\right)$ to a Lipschitz function $u_{0}$. We will call such a function $u_{0}$ a blowup of $u$ at $x_{0}$. In fact, there is a more general construction which allows us to take rescalings of different minimizers $u_{k}$ and even allows the exponent $p=p_{k}$ to vary.

Theorem 2.3 (Blowup with variable $p$ ). Let $1<p<\infty$ and suppose we have a sequence of exponents $1<p_{k}<\infty, k=1,2, \ldots$, such that $p_{k} \rightarrow p$. Let $u_{k}$ be a minimizer of $J_{p_{k}}$ in $B_{1}$ such that $0 \in \Gamma\left(u_{k}\right)$. Then for any sequence $\lambda_{k} \rightarrow 0$ we can find a subsequence so that the rescalings

$$
u_{k, \lambda_{k}}(x)=\frac{u_{k}\left(\lambda_{k} x\right)}{\lambda_{k}}, \quad x \in B_{1 / \lambda_{k}}
$$


converge in $L_{\text {loc }}^{\infty}\left(\mathbb{R}^{n}\right)$ to a continuous function $u_{0}$ in $\mathbb{R}^{n}$, which we call a blowup. Moreover, every such blowup $u_{0}$ is a global minimizer of $J_{p}$, i.e. a minimizer on every bounded open set, and $0 \in \Gamma\left(u_{0}\right)$.

\subsection{Flatness and regularity.}

Definition 2.4. Let $0 \leq \sigma_{+}, \sigma_{-} \leq 1$ and $\tau>0$. We say that $u$ is of the flatness class $F^{p}\left(\sigma_{+}, \sigma_{-} ; \tau\right)$ in the ball $B_{\rho}$ if $u$ is a minimizer of $J_{p}$ in $B_{\rho}, 0 \in \Gamma(u)$, and

$$
\begin{array}{ll}
u(x)=0 & \text { for } x_{n} \geq \sigma_{+} \rho, \\
u(x) \geq-\left(x_{n}+\sigma_{-} \rho\right) & \text { for } x_{n} \leq-\sigma_{-} \rho, \\
|\nabla u| \leq 1+\tau & \text { in } B_{\rho} .
\end{array}
$$

More generally, changing the direction $e_{n}$ by $\nu$ and the origin by $x_{0}$ in the definition above, we obtain the definition of the flatness class $F^{p}\left(\sigma_{+}, \sigma_{-} ; \tau\right)$ in $B_{\rho}\left(x_{0}\right)$ in direction $\nu$.

Theorem 2.5 (Flatness implies regularity). Let $u$ be a minimizer of $J_{p}$ in $B_{1}$, $0 \in \Gamma(u)$ and $p \in[1+\mu, 1+1 / \mu], 0<\mu<1$. Then there exist positive constants $\alpha, \beta, \sigma_{0}, \tau_{0}$ depending only on $n$ and $\mu$ such that

$$
\begin{aligned}
& \text { if } u \in F^{p}(\sigma, 1 ; \infty) \text { in } B_{\rho} \text { in some direction with } \sigma \leq \sigma_{0}, \rho \leq \tau_{0} \sigma^{2 / \beta}, \\
& \text { then } \Gamma(u) \cap B_{\rho / 4} \text { is a } C^{1, \alpha} \text { hypersurface. }
\end{aligned}
$$

Remark 2.6. A theorem of Kinderlehrer, Nirenberg and Spruck KNS78 then implies that $\Gamma(u) \cap B_{\rho / 4}$ is an analytic hypersurface. Their results are applicable, since condition (1.2) is satisfied in the $C^{1}$ sense on that portion of $\Gamma(u)$ which makes the $p$-Laplacian uniformly elliptic in a neighborhood.

The proof of the preceding theorem is obtained by iteration from the following lemma, which is really the core of the argument.

Lemma 2.7 (Improvement of flatness). Let $u$ be a minimizer of $J_{p}$ in $B_{1}, 0 \in \Gamma(u)$ and $p \in[1+\mu, 1+1 / \mu], 0<\mu<1$. Then for any $\theta>0$ there exist constants $\sigma_{\theta}=\sigma(\theta, \mu, n)>0, c_{\theta}=c(\theta, \mu, n)>0$ and $C=C(n, \mu)>0$ such that if

$$
u \in F^{p}(\sigma, 1 ; \tau) \text { in } B_{\rho} \text { in direction } \nu,
$$

with $\sigma \leq \sigma_{\theta}$ and $\tau \leq c_{\theta} \sigma^{2}$, then

$$
u \in F^{p}\left(\theta \sigma, \theta \sigma ; \theta^{2} \tau\right) \text { in } B_{\bar{\rho}} \text { in direction } \bar{\nu}
$$

for some $\bar{\rho}, \bar{\nu}$ with $c_{\theta} \rho \leq \bar{\rho} \leq \rho / 4$ and $|\nu-\bar{\nu}| \leq C \sigma$.

\section{Proof OF THE MAIN RESUlt}

Basically, the proof is a combination of the following three ingredients: (i) flatness implies regularity (Theorem 2.5), (ii) blowup with variable $p$ (Theorem 2.3), and (iii) a theorem on global minimizers of $J_{p}$ for $p \in \mathcal{R}(n)$, similar to that of Bernstein and Simons in minimal surface theory [Sim67], which we state next. Recall that a global minimizer is a function in $W_{\text {loc }}^{1, p}\left(\mathbb{R}^{n}\right) \cap L_{\text {loc }}^{\infty}\left(\mathbb{R}^{n}\right)$, which is a minimizer of $J_{p}$ on every bounded open set of $\mathbb{R}^{n}$.

Theorem 3.1 (Bernstein-type). Let $p \in \mathcal{R}(n)$. Then every global minimizer $u$ of $J_{p}$ with $0 \in \Gamma(u)$ is a halfspace solution; i.e., there exists a direction e such that $u(x)=(x \cdot e)^{+}$for every $x \in \mathbb{R}^{n}$.

We will need the following intermediate result to establish Theorem 3.1 . 
Lemma 3.2. Let $u$ be a global minimizer of $J_{p}$ and $0 \in \Gamma(u)$. Then

$$
|\nabla u| \leq 1 \quad \text { in } \quad\{u>0\} .
$$

Proof. We will use a particular case of Theorem 7.1 in DP05: if $u$ is a minimizer of $J_{p}$ in $B_{1}$ and $0 \in \Gamma(u)$, then

$$
\sup _{B_{r}}|\nabla u| \leq 1+C r^{\alpha}
$$

for any $0<r \leq 1 / 2$ with constants $C>0,0<\alpha<1$, depending only on $n$ and $p$. Now, applying this inequality to rescalings

$$
u_{0, \lambda}(x)=\frac{u(\lambda x)}{\lambda}, \quad x \in \mathbb{R}^{n}
$$

we will obtain

$$
\sup _{B_{r}}|\nabla u| \leq 1+C(r / \lambda)^{\alpha}
$$

for any $r \leq \lambda / 2$. Letting $\lambda \rightarrow \infty$, we complete the proof of the lemma.

Proof of Theorem 3.1. Let $u$ be a global minimizer of $J_{p}$ with $0 \in \Gamma(u)$. Consider the sequence of rescalings

$$
u_{n}(x)=u_{0, n}(x)=\frac{u(n x)}{n}, \quad n=1,2, \ldots,
$$

which are also global minimizers of $J_{p}$. From the uniform Lipschitz continuity, or better yet from Lemma 3.2. we can extract a subsequence $n_{k} \rightarrow \infty$ such that $u_{n_{k}}$ converges locally uniformly to a global minimizer $u_{\infty}$ (Theorem 2.3). From the nondegeneracy theorem (Theorem 2.2), we have that

$$
0 \in \Gamma\left(u_{\infty}\right) .
$$

Since $p \in \mathcal{R}(n)$, by definition of the class $\mathcal{R}(n), 0$ is a regular free boundary point for $u_{\infty}$ and therefore there exists a ball $B \subset\left\{u_{\infty}=0\right\}$ touching $\Gamma\left(u_{\infty}\right)$ at 0 . Rotating the coordinate system, we may assume that $B=B_{r}\left(r e_{n}\right)$. But this implies that for any $\sigma>0$,

$$
u_{\infty} \in F^{p}(\sigma, 1 ; 0) \text { in } B_{r(\sigma)}
$$

if $r(\sigma)$ is chosen sufficiently small. Note that the parameter $\tau=0$ in the flatness class above, since by Lemma 3.2 we have $\left|\nabla u_{\infty}\right| \leq 1$ in $\mathbb{R}^{n}$.

Next, from the nondegeneracy theorem (Theorem 2.2) we will have

$$
u_{n_{k}} \in F^{p}(3 \sigma, 1 ; 0) \quad \text { in } B_{r(\sigma) / 2},
$$

for $k \geq k(\sigma)$. The latter is equivalent to

$$
u \in F^{p}(3 \sigma, 1 ; 0) \quad \text { in } B_{r(\sigma) n_{k} / 2},
$$

for $k \geq k(\sigma)$. The rest of the proof is based on a flatness improvement argument. Fix a certain $0<\theta<1 / 3$ and assume that $\sigma<\sigma_{\theta}$. Now take $k$ large and apply Lemma 2.7 with $\rho=r(\sigma) n_{k} / 2$ iteratively $N$ times. We will obtain a sequence of radii $\bar{\rho}_{j}, j=0,1, \ldots, N$, with

$$
\bar{\rho}_{0}=\rho=r(\sigma) n_{k} / 2, \quad c_{\theta} \bar{\rho}_{j} \leq \bar{\rho}_{j+1} \leq \bar{\rho}_{j} / 4
$$

such that

$$
u \in F^{p}\left(3 \theta^{j} \sigma, 3 \theta^{j} \sigma ; 0\right) \quad \text { in } B_{\bar{\rho}_{j}} \text { in some direction. }
$$


Now, fix large $R>0$, and take $n_{k}$ with $k \geq k(\sigma)$ such that $\bar{\rho}_{0}=\rho(\sigma) n_{k} / 2>R$. Next, choose the number of steps $N$ in the iteration above so that

$$
\bar{\rho}_{N} \leq R \leq \bar{\rho}_{N-1} .
$$

Putting $\bar{R}=\bar{\rho}_{N}$, we will basically obtain that for any $0<\sigma<\sigma_{\theta}$ there exists $c_{\theta} R \leq \bar{R} \leq R$ such that

$$
u \in F^{p}(\sigma, \sigma ; 0) \text { in } B_{\bar{R}} \text { in some direction. }
$$

Consequently,

$$
u \in F^{p}\left(\sigma / c_{\theta}, \sigma / c_{\theta} ; 0\right) \text { in } B_{c_{\theta} R} \text { in some direction; }
$$

i.e. the free boundary of $u$ is as flat in $B_{c_{\theta} R}$ as we wish. Hence, letting $\sigma \rightarrow 0$ and then $R \rightarrow \infty$, we obtain that $u$ is necessarily a halfspace solution.

We are now ready to prove the main result.

Proof of the main result (Theorem [1.2).

Step 1. We start with the claim that for any $\sigma>0$ there exists $\epsilon(\sigma)>0$ and $r(\sigma)>0$ such that if $u$ is a minimizer of $J_{p}$ in $B_{1}$ of $\mathbb{R}^{n}$ with $0 \in \Gamma(u)$, then

$$
u \in F^{p}(\sigma, 1 ; \infty) \text { in } B_{r} \text { in some direction, }
$$

provided

$$
\left|p-p_{0}\right|<\epsilon(\sigma), \quad 0<r \leq r(\sigma) .
$$

This will follow by a blowup argument, combined with the Bernstein-type theorem. Indeed, assuming the contrary, let $u_{n}$ be a minimizer of $J_{p_{n}}$ in $B_{1}$ with $0 \in \Gamma\left(u_{n}\right)$, $p_{n} \rightarrow p_{0}$ and suppose that for some $r_{n} \rightarrow 0+$

$$
u_{n} \notin F^{p_{n}}(\sigma, 1 ; \infty) \text { in } B_{r_{n}} \text { in any direction. }
$$

Then consider the rescalings

$$
\tilde{u}_{n}(x)=\frac{u_{n}\left(r_{n} x\right)}{r_{n}}, \quad x \in B_{1 / r_{n}},
$$

which are minimizers of $J_{p_{n}}$ in $B_{1 / r_{n}}$. Over a subsequence, they converge locally uniformly to a global minimizer $u_{0}$ of $J_{p_{0}}$; see Theorem 2.3. Since we assume $p_{0} \in \mathcal{R}(n)$, by Theorem 3.1 we have that $u_{0}$ is a halfspace solution; i.e.,

$$
u_{0}(x)=(x \cdot e)^{+}, \quad x \in \mathbb{R}^{n},
$$

for a unit vector $e$. The nondegeneracy theorem, Theorem 2.2. now implies that

$$
\tilde{u}_{n}=0 \quad \text { on } \overline{B_{1}} \cap\{x \cdot e \leq-\sigma\}
$$

for sufficiently large $n$. But this exactly means $\tilde{u}_{n} \in F^{p_{n}}(\sigma, 1 ; \infty)$ in direction $-e$, or equivalently,

$$
u_{n} \in F^{p_{n}}(\sigma, 1 ; \infty) \text { in } B_{r_{n}} \text { in direction }-e,
$$

contrary to our assumption.

Step 2. Now let $\sigma_{0}, \tau_{0}$, and $\beta$ be as in Theorem 2.5 and choose $0<\sigma_{1}<\sigma_{0}$. Now, let $\epsilon\left(\sigma_{1}\right)$ and $r\left(\sigma_{1}\right)$ be as in Step 1 above for $\sigma=\sigma_{1}$ and let

$$
r_{1}=\min \left\{r\left(\sigma_{1}\right), \tau_{0} \sigma_{1}^{2 / \beta}\right\} .
$$

Then, by the argument in Step 1 , if $u$ is a minimizer of $J_{p}$ in $B_{1}$ with $0 \in \Gamma(u)$ and

$$
\left|p-p_{0}\right|<\epsilon\left(\sigma_{1}\right)
$$


then

$$
u \in F^{p}\left(\sigma_{1}, 1 ; \infty\right) \text { in } B_{r_{1}} \text { in some direction. }
$$

Furthermore, the construction above guarantees that the conditions of Theorem 2.5 are satisfied, and therefore we obtain that $B_{r_{1} / 4} \cap \Gamma(u)$ is a $C^{1, \alpha}$ for some $0<\alpha<1$ and thus analytic by a theorem of Kinderlehrer, Nirenberg, and Spruck [KNS78.

Step 3. Finally, we have already remarked that the regularity of the free boundary is a local property and because of the scaling, Step 2 essentially completes the proof. Let us make this more precise. Let $u$ be a minimizer of $J_{p}$ with $\left|p-p_{0}\right|<\epsilon\left(\sigma_{1}\right)$ in an open set $\Omega$ and let $x_{0} \in \Gamma(u)$ be arbitrary. Then $B_{\rho}\left(x_{0}\right) \Subset \Omega$ for some $\rho>0$ and the rescaling

$$
u_{x_{0}, \rho}(x)=\frac{u\left(\rho x+x_{0}\right)}{\rho}, \quad x \in B_{1}
$$

is a minimizer of $J_{p}$ in $B_{1}$ with $0 \in \Gamma\left(u_{x_{0}, \rho}\right)$. Then, by Step 2 above, $B_{r_{1} / 4} \cap \Gamma\left(U_{x_{0}, \rho}\right)$ is analytic and, scaling back, we obtain that $B_{\rho r_{1} / 4}\left(x_{0}\right) \cap \Gamma(u)$ is analytic. This completes the proof of the theorem.

\section{REFERENCES}

[AC81] H. W. Alt and L. A. Caffarelli, Existence and regularity for a minimum problem with free boundary, J. Reine Angew. Math. 325 (1981), 105-144. MR618549 (83a:49011)

[CJK04] Luis A. Caffarelli, David Jerison, and Carlos E. Kenig, Global energy minimizers for free boundary problems and full regularity in three dimensions, Noncompact problems at the intersection of geometry, analysis, and topology, Contemp. Math., 350, Amer. Math. Soc., Providence, RI, 2004, pp. 83-97. MR2082392 (2005e:35258)

[DP05] Donatella Danielli and Arshak Petrosyan, A minimum problem with free boundary for a degenerate quasilinear operator, Calc. Var. Partial Differential Equations 23 (2005), no. 1, 97-124. MR2133664 (2006c:35303)

[DP06] - Full regularity of the free boundary in a Bernoulli-type problem in two dimensions, Math. Res. Lett. 13 (2006), no. 4, 667-681. MR2250499 (2007f:35306)

[DSJ05] Daniela De Silva and David Jerison, A singular energy minimizing free boundary (2005), preprint.

[KNS78] David Kinderlehrer, Louis Nirenberg, and Joel Spruck, Régularité dans les problèmes elliptiques à frontière libre, C. R. Acad. Sci. Paris Sér. A-B 286 (1978), no. 24, A1187A1190 (French, with English summary). MR501110 (80a:35050)

[MW06] Sandra Martínez and Noemi Wolanski, A minimum problem with free boundary in Orlicz spaces (2006), preprint.

[Sim67] James Simons, Minimal cones, Plateau's problem, and the Bernstein conjecture, Proc. Nat. Acad. Sci. U.S.A. 58 (1967), 410-411. MR0216387(35:7221)

[Wei99] Georg Sebastian Weiss, Partial regularity for a minimum problem with free boundary, J. Geom. Anal. 9 (1999), no. 2, 317-326. MR1759450(2001b:49053)

Department of Mathematics, Purdue University, West Lafayette, Indiana 47907

E-mail address: arshak@math.purdue.edu 